Enferm Bras 2019;18(4);552-60

https://doi.org/10.33233/eb.v18i4.2756

\title{
ARTIGO ORIGINAL \\ Perfil social-profissional de enfermeiros e médicos da Atenção Primária à Saúde de uma microrregião geográfica
}

Lais Soares Santos ${ }^{\star}$, Tassiana Elena de Souza (In memoriam) ${ }^{\star *}$, Carla Elisia Souza ${ }^{\star \star}$, Mickaela Cunha Monteiro**, Mara Rúbia Maciel Cardoso Prado***, Pedro Paulo do Prado Júnior ${ }^{\star \star *}$, Lilian Fernandes Arial Ayres ${ }^{\star * \star}$, Camila Mendes dos Passos ${ }^{\star * \star *}$

*Graduanda em Enfermagem pelo Departamento de Medicina e Enfermagem (DEM),

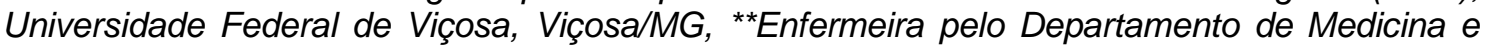
Enfermagem (DEM), Universidade Federal de Viçosa, Viçosa/MG, ${ }^{* * *}$ Professor (a) do Departamento de Medicina e Enfermagem (DEM), Universidade Federal de Viçosa, Viçosa/MG, ****Professor do Departamento de Medicina e Enfermagem (DEM), Universidade Federal de Viçosa, Viçosa/MG, Doutoranda em Enfermagem pela Universidade Federal de Minas Gerais, Belo Horizonte/MG

Recebido em 28 de janeiro de 2019; aceito em 22 de maio de 2019.

Correspondência: Camila Mendes dos Passos, Departamento de Medicina e Enfermagem, Universidade Federal de Viçosa, Av. Peter Henry Rolfs, s/n, Campus Universitário 36570-900 Viçosa MG

Camila Mendes dos Passos: camilampassos@yahoo.com.br

Lais Soares Santos: lais.s.soares@ufv.br

Carla Elisia Souza: souza.carlaelisia@gmail.com

Mickaela Cunha Monteiro: mickaelacunham@gmail.com

Mara Rúbia Maciel Cardoso Prado: mara.prado@ufv.br

Pedro Paulo do Prado Júnior: pedro.prado@ufv.br

Lilian Fernandes Arial Ayres: liliayresenf@yahoo.com.br

\section{Resumo}

Introdução: Profissionais de saúde de nível superior são protagonistas das ações pactuadas na Atenção Primária à Saúde, imprimindo e determinando diferentes direções no processo de trabalho em saúde. Enfermeiros e médicos, atuando em equipe, são atores centrais no processo de trabalho em saúde em nível primário. Objetivo: Identificar o perfil social-profissional de enfermeiros e médicos da Atenção Primária à Saúde de uma microrregião geográfica no estado de Minas Gerais, considerando a sua atuação no pré-natal de baixo risco. Métodos: Estudo transversal, quantitativo e de caráter censitário, realizado com 135 enfermeiros e médicos que atuam na Atenção Primária à Saúde da microrregião geográfica. Os dados foram coletados por entrevista estruturada. Medidas de frequência absoluta e relativa foram utilizadas para descrição da população. O teste qui-quadrado de Pearson foi utilizado para verificar a diferença no perfil sócio profissional entre os profissionais. Considerou-se nível de significância de $p<0,05$. Resultados: Participaram do estudo 74 enfermeiros (54,8\%) e 61 médicos (45,2\%). Os profissionais de saúde investigados foram predominantemente jovens, mulheres, pós-graduados, com pouco tempo de experiência profissional e de atuação na unidade de trabalho. Conclusão: Os enfermeiros e médicos possuem perfil social-profissional semelhante, com diferença principalmente na instituição de graduação (pública e privada).

Palavras-chave: atenção primária à saúde, saúde da família, pessoal de saúde.

\section{Abstract}

Social-professional profile of nurses and doctors in the primary health care network in a geographic micro-region

Introduction: Graduated health professionals are protagonists of the actions agreed in the Primary Attention to Health, determining different directions in the work process in health. Nurses and physicians, acting as a team, are central actors in the health work process at the primary level. Objective: To identify the social-professional profile of primary health care nurses and doctors in a geographic micro-region in the state of Minas Gerais, considering its performance in low risk 
prenatal care. Methods: A cross-sectional, quantitative and census-based study was carried out with 135 nurses and physicians working at the primary health care in the geographic micro-region. Data were collected by structured interviews. Absolute and relative frequency measurements were used to describe the population. Pearson's chi-square test was used to verify the difference in the socio-professional profile among the professionals. A significance level of $p<0.05$ was considered. Results: 74 nurses $(54.8 \%)$ and 61 physicians $(45.2 \%)$ participated in the study. The health professionals investigated were predominantly young, female, post-graduate, with little time of professional and work unit experience. Conclusion: Nurses and physicians have similar social-professional profile, with a difference mainly in the undergraduate institution (public and private).

Key-words: primary health care, family health, health personnel.

\section{Resumen \\ Perfil social-profesional de enfermeros y médicos en la red de atención primaria de salud en una micro-región geográfica}

Introducción: Los profesionales de salud de nivel superior son protagonistas de las acciones pactadas en la Atención Primaria a la Salud, imprimiendo y determinando diferentes direcciones en el proceso de trabajo en salud. Enfermeros y médicos, actuando en equipo, son actores centrales en el proceso de trabajo en salud a nivel primario. Objetivo: Identificar el perfil socialprofesional de enfermeros y médicos de la Atención Primaria a la Salud de una microrregión geográfica en el estado de Minas Gerais, considerando su actuación en el prenatal de bajo riesgo. Métodos: Estudio transversal, cuantitativo y de carácter censitario, realizado con 135 enfermeros y médicos que actúan en la Atención Primaria a la Salud de la microrregión geográfica. Los datos fueron recolectados por una entrevista estructurada. Se utilizaron medidas de frecuencia absoluta y relativa para la descripción de la población. La prueba qui-cuadrada de Pearson fue utilizada para verificar la diferencia en el perfil socio profesional entre los profesionales. Se consideró un nivel de significancia de $p<0,05$. Resultados: Participaron del estudio 74 enfermeros $(54,8 \%)$ y 61 médicos $(45,2 \%)$. Los profesionales de salud investigados fueron predominantemente jóvenes, mujeres, postgraduados, con poco tiempo de experiencia profesional y de actuación en la unidad de trabajo. Conclusión: Se concluyó que los enfermeros y médicos poseen perfil social-profesional semejante, con diferencia principalmente en la institución de graduación (pública y privada).

Palabras-clave: atención primaria a la salud, salud de la familia, personal de salud.

Introdução

A atenção à saúde gratuita e de qualidade são um dos principais desafios do Sistema Único de Saúde (SUS) brasileiro. Esse sistema está pautado nos princípios da integralidade, universalidade, equidade, regionalização, hierarquização e participação social [1]. Para tanto, é primordial a ampliação do acesso e a qualificação da Atenção Primária à Saúde (APS), orientadora da rede de serviços de saúde [2]. A APS possui caráter resolutivo frente a maior parte das demandas e necessidade de saúde da população, podendo impactar direta ou indiretamente nos determinantes e condicionantes de saúde das populações por meio da integralização do cuidado [3].

O desenvolvimento de uma atenção integral pautada em ações de promoção, proteção e recuperação pode ser alcançado por meio de um modelo assistencial organizado em rede e por uma estratégia que prioriza a assistência voltada para a família e o indivíduo, entendendo-o como parte de uma sociedade [3]. A Estratégia Saúde da Família (ESF) torna-se um importante instrumento capaz de interferir na lógica da oferta a partir da demanda, promovendo maior acesso, mudança do modelo assistencial e garantindo qualidade ao SUS [4,5]. Sobretudo, mudanças na APS são necessárias, principalmente no modo de operar os processos de trabalho em saúde, transformando a dinâmica de trabalho médico-centrado em trabalho multiprofissional [6]. A equipe mínima de saúde, formada por enfermeiro, médico, auxiliar de enfermagem e agentes comunitários de saúde, ocupa lugar de destaque na organização das práticas de saúde e no desenvolvimento das ações em consonância com este modelo proposto [2].

Os profissionais de saúde de nível superior assumem caráter de protagonismo diante das ações pactuadas, imprimindo e determinando diferentes direções técnicas, éticas e políticas no processo de trabalho em saúde. É preciso reconhecer, portanto, a importância do perfil de enfermeiros e médicos que atuam na APS [7]. Cabe ao profissional vivenciar a realidade da comunidade com mais frequência e agir nesse território, considerando o indivíduo como sujeito 
social [8]. Desse modo, o conhecimento teórico é capaz de transformar as condições de saúde do sujeito, por meio do trabalho multiprofissional e ações de enfoques familiares, com perspectivas generalistas [8].

A APS requer profissionais de saúde com saberes ampliados, que possuem habilidades e competências técnicas, que desenvolvam também além das dimensões políticas, a gestão do trabalho em saúde, assumindo a gestão do seu próprio trabalho [9]. Profissionais que buscam conhecimento, aprimoramento, entendendo que a população está em intensa evolução, e as tecnologias para o cuidado estão cada vez mais aprimoradas e prontas para serem utilizadas [9].

Enfermeiros e médicos da família assumem, por vezes, um papel de generalistas, cabendo a eles considerar as diversidades dos indivíduos, das famílias e dos ambientes em que vivem que por sua vez determinam sua saúde [10]. Entretanto, esses profissionais também são habilitados e atuam em ações específicas como a assistência ao pré-natal de baixo risco [11]. $O$ cuidado pré-natal é uma das atividades primordiais no cenário da APS por se ater ao trabalho multiprofissional e em equipe, que quando qualificado é capaz de melhorar indicadores de saúde infantil e maternos [12].

Frente ao exposto, emergiu o interesse para realização do estudo. Considerando a importância dos profissionais de saúde, enfermeiros e médicos da APS, atuando no trabalho em equipe, enquanto atores centrais no processo de trabalho em saúde em nível primário. $\mathrm{E}$, além disso, por se desconhecer o perfil desses profissionais no cenário investigado, bem como a qualificação desses profissionais em relação à assistência primária à saúde. Nesse contexto, objetivou-se identificar o perfil social-profissional de enfermeiros e médicos da atenção primária à saúde de uma microrregião geográfica no estado de Minas Gerais, considerando a sua atuação no pré-natal de baixo risco. Acredita-se que o perfil desses profissionais seja semelhante entre si, porém com diferenças entre perfis de profissionais de saúde, em outros cenários já conhecidos, que atuam em regiões metropolitanas.

\section{Material e métodos}

A investigação é derivada de um estudo mais abrangente intitulado "Conhecimentos, atitudes e prática de enfermeiros e médicos sobre a assistência pré-natal de baixo risco nos municípios da microrregião Viçosa/MG". Trata-se de um estudo transversal, descritivo, de abordagem quantitativa e de caráter censitário.

O estudo foi realizado na microrregião geográfica de Viçosa, situada na região da Zona da Mata do Estado de Minas Gerais (MG), formada por 20 municípios, sendo eles: Alto Rio Doce, Amparo da Serra, Araponga, Brás Pires, Cajuri, Canaã, Cipotânea, Coimbra, Ervália, Lamim, Paula Cândido, Pedra do Anta, Piranga, Porto Firme, Presidente Bernardes, Rio Espera, São Miguel do Anta, Senhora de Oliveira, Teixeiras e Viçosa [13].

A população de estudo foi composta por profissionais enfermeiros e médicos, que compõem todas as 83 equipes de ESF dos municípios da microrregião Viçosa/MG. A escolha destes profissionais se justifica pela recomendação do Ministério da Saúde, na qual as equipes de saúde da família são formadas obrigatoriamente por esses dois profissionais de nível superior, enfermeiro e médico, adicionado a outros profissionais de nível médio como auxiliar de enfermagem e agente comunitário de saúde (ACS) [14]. Essa população foi estimada por meio do número mínimo de profissionais médicos e enfermeiros previstos a partir do quantitativo de equipes de Saúde da Família. Para tanto, previu-se a participação de 163 participantes de pesquisa, destes 83 enfermeiros e 80 médicos.

A coleta de dados ocorreu no período de 23 de maio de 2016 a 15 de agosto de 2016 . As entrevistas foram realizadas com o auxílio de um questionário estruturado, autoaplicável, com duração média de 60 minutos. Em sua maioria, foram previamente agendadas, respeitando 0 melhor horário e localidade para o participante da pesquisa. Antes do início de cada aplicação, realizava-se a leitura do Termo de Consentimento Livre e Esclarecido (TCLE) para compreensão dos objetivos e procedimentos do estudo e assinatura do mesmo. Durante essa etapa, também foram incluídos no estudo, residentes de medicina e de enfermagem que atuavam nas unidades participantes no período da coleta de dados. Ao final, foram excluídos 14 profissionais por se encontrarem no período de férias ou de licença ou não serem localizados no dia da coleta. Outros 20 participantes, por se recusarem participar do estudo. A população final entrevistada foi constituída por 74 enfermeiros, 55 médicos e 06 residentes de medicina. Somados totalizou-se uma amostra de 135 profissionais, o equivalente a $82,82 \%$ da população esperada.

Antecedendo a fase de coleta dos dados, fez-se a construção do questionário, que abrangeu duas etapas sequenciais. A primeira delas consistiu na elaboração do instrumento 
baseando-se nas recomendações, diretrizes e fundamentos teóricos a partir de livros-textos clássicos sobre a temática, além de manuais e protocolos oficiais do Ministério da Saúde $[15,16]$. As perguntas que compuseram $o$ instrumento abordaram as características sociais $e$ profissionais, bem como os conhecimentos, atitudes e práticas na linha de cuidado da assistência pré-natal.

A segunda etapa fundamentou-se na validação de aparência e conteúdo do questionário, a qual foi realizada por três pareceristas com expertise sobre a temática, docentes do curso de Enfermagem de três instituições de ensino superior federal de Minas Gerais. As assertivas do instrumento foram pontuadas de acordo com o grau de importância: 0 - desnecessário, 1 - regular, 2 - bom, 3 - ótimo. Ao final, as questões que obtiveram pontuação menor que cinco foram retiradas do instrumento, sendo consideradas irrelevantes para o estudo. Neste processo foram excluídas 02 questões do instrumento completo.

Neste estudo foram consideradas e utilizadas apenas as questões referentes à caracterização social e profissional dos participantes. Para a análise dos dados, construiu-se um banco de dados no programa Excel, versão 2013, no qual foi realizada a entrada dupla com posterior análise de erros e inconsistências. O banco foi exportado para o programa SPSS (Statistical Package for the Social Sciences), versão 22.0, onde foi realizada a organização, recodificação e análise. Procedeu-se a análise descritiva da população de estudo. Para as variáveis contínuas usou-se o teste Kolmogorov-Smirnov para testar a normalidade dos dados. Após isso, decidiu-se apresentar como medida de tendência central, a mediana e a amplitude interquartil (25-75) como medida de dispersão. Para as variáveis categóricas utilizaram-se medidas de frequência absoluta e relativa para descrição das mesmas. O teste Qui-quadrado de Pearson foi utilizado para verificar a diferença no perfil sócio-profissional entre médicos e enfermeiros. Para todas as análises foi considerado nível de significância de $p<0,05$ na verificação de diferenças entre os grupos.

Por se tratar de pesquisa envolvendo seres humanos, o estudo foi submetido e aprovado pelo Comitê de Ética em Pesquisa da Universidade Federal de Viçosa (CEP/UFV) em 15 de fevereiro de 2016, apresentando parecer nํㅜ 1.408.471.

Resultados

Participaram do estudo 135 profissionais de saúde, sendo 74 enfermeiros $(54,8 \%)$ e 61 médicos $(45,2 \%)$ (Tabela I). Quase a totalidade deles (97\%) atua em equipes de saúde da família na rede de APS dos municípios participantes. Esses profissionais possuem uma mediana de idade de 31 anos $(27,0-39,25)$, de tempo de experiência profissional de 5 anos $(2,0-9,0)$ e de tempo de atuação na unidade de apenas 2 anos $(0,5-4,0)$.

Identificou-se que os enfermeiros possuem em sua maioria idade de até 30 anos $(40,6 \%)$, são do sexo feminino $(83,8 \%)$, estudaram em escola privada $(73,0 \%)$, possuem pós-graduação $(62,2 \%)$, experiência profissional de 1 ano a 6 anos (50,7\%) e atuam de 1 a 4 anos na ESF $(43,1 \%)$. Em relação à capacitação sobre pré-natal, $52,7 \%$ referiram nunca ter realizado, embora $68,9 \%$ dos enfermeiros classificaram o conhecimento sobre assistência pré-natal como "bom". Mais da metade deles $(57,5 \%)$ alegam uma boa capacidade de realização da consulta, porém em torno de $45 \%$ realizam poucas vezes ou nunca esse tipo de atendimento (Tabela I).

Em relação ao profissional médico, $50 \%$ possuem idade de até 30 anos, 50,8\% são do sexo feminino, $53,3 \%$ se formaram em instituições públicas e $55,7 \%$ informaram ter pósgraduação, 43,1\% possuem de 1 a 6 anos de experiência profissional e 50,8\% trabalham há apenas um ano ou menos na ESF. Em relação à assistência pré-natal, $67,2 \%$ afirmam nunca ter realizado capacitação, se autoavaliaram com "boa" capacidade de realizar a consulta e com "bom" conhecimento sobre a temática (assistência pré-natal). Metade dos profissionais médicos sempre realiza $o$ atendimento (Tabela I).

Conforme apontado na Tabela I, na análise do perfil social e profissional dos enfermeiros e médicos há diferença entre os grupos com significância estatística $(p<0,05)$ em relação à idade, à instituição de graduação, ao tempo de atuação na ESF, à classificação da capacidade de realizar o pré-natal e à frequência da realização das consultas. 
Tabela I - Distribuição do número de enfermeiros e médicos de acordo com as características sociais e profissionais. Microrregião geográfica de Minas Gerais, 2016.

\begin{tabular}{|c|c|c|c|c|c|}
\hline \multirow[t]{2}{*}{ Variáveis } & \multicolumn{2}{|c|}{ Enfermeiros } & \multicolumn{2}{|c|}{ Médicos } & \multirow[t]{2}{*}{ Valor de $p(1)$} \\
\hline & $N$ & $(\%)$ & $N$ & $(\%)$ & \\
\hline Idade & & & & & 0,174 \\
\hline Até 30 anos & 26 & 40,6 & 27 & 50,0 & \\
\hline 31 a 40 anos & 22 & 34,4 & 16 & 29,6 & \\
\hline 41 a 50 anos & 12 & 18,8 & 4 & 7,4 & \\
\hline Acima de 50 anos & 4 & 6,3 & 7 & 13,0 & \\
\hline Sexo & & & & & $<0,001^{*}$ \\
\hline Feminino & 62 & 83,8 & 31 & 50,8 & \\
\hline Masculino & 12 & 16,2 & 30 & 49,2 & \\
\hline Instituição de graduação & & & & & $0,002^{*}$ \\
\hline Pública & 20 & 27,0 & 32 & 53,3 & \\
\hline Privada & 54 & 73,0 & 28 & 46,7 & \\
\hline Escolaridade & & & & & 0,450 \\
\hline Graduação & 28 & 37,8 & 27 & 44,3 & \\
\hline Pós-graduação & 46 & 62,2 & 34 & 55,7 & \\
\hline Área de abrangência da equipe & & & & & 0,365 \\
\hline Zona urbana & 21 & 28,4 & 12 & 19,7 & \\
\hline Zona rural & 25 & 33,8 & 27 & 44,3 & \\
\hline Ambas & 28 & 37,8 & 22 & 36,1 & \\
\hline Experiência profissional & & & & & $<0,001^{*}$ \\
\hline 1 ano ou menos & 5 & 7,0 & 16 & 27,6 & \\
\hline 1 a 6 anos & 36 & 50,7 & 25 & 43,1 & \\
\hline 6 a 11 anos & 23 & 32,4 & 1 & 1,7 & \\
\hline 11 a 16 anos & 6 & 8,5 & 3 & 5,2 & \\
\hline Mais de 16 anos & 1 & 1,4 & 13 & 22,4 & \\
\hline Atuação na unidade & & & & & $0,028^{*}$ \\
\hline 1 ano ou menos & 20 & 27,8 & 30 & 50,8 & \\
\hline 1 a 4 anos & 31 & 43,1 & 21 & 35,6 & \\
\hline 4 a 7 anos & 14 & 19,4 & 4 & 6,8 & \\
\hline Mais de 7 anos & 7 & 9,7 & 4 & 8,2 & \\
\hline Realizou capacitação sobre pré-natal & & & & & 0,088 \\
\hline Sim & 35 & 47,3 & 20 & 32,8 & \\
\hline Não & 59 & 52,7 & 41 & 67,2 & \\
\hline Classificação do conhecimento sobre pré-natal & & & & & 0,077 \\
\hline Muito bom & 5 & 6,8 & 11 & 18 & \\
\hline Bom & 51 & 68,9 & 41 & 67,2 & \\
\hline Regular & 18 & 24,3 & 9 & 14,8 & \\
\hline Classificação da capacidade de realizar pré-natal & & & & & $<0,001^{*}$ \\
\hline Muito bom & 6 & 8,2 & 14 & 23,0 & \\
\hline Bom & 42 & 57,5 & 41 & 67,2 & \\
\hline Regular ou ruim & 25 & 34,2 & 6 & 9,8 & \\
\hline Frequência que realiza consulta pré-natal & & & & & $<0,001^{*}$ \\
\hline Sempre & 11 & 15,3 & 30 & 50,0 & \\
\hline Quase sempre & 12 & 16,7 & 7 & 11,7 & \\
\hline Algumas vezes & 16 & 22,2 & 13 & 21,7 & \\
\hline Poucas vezes & 22 & 30,6 & 6 & 10,0 & \\
\hline Nunca & 11 & 15,3 & 4 & 6,7 & \\
\hline Total & 74 & 54,8 & 61 & 45,2 & \\
\hline
\end{tabular}

\section{Discussão}

A predominância do sexo feminino em ambas as profissões corrobora demais estudos

No que diz respeito aos enfermeiros, reafirma a história e a trajetória da profissão, embora tenha sido observado um aumento do quantitativo masculino nos últimos anos nesta categoria [21]. Em relação aos médicos, as mulheres também representaram a maioria, entretanto houve uma diferença pequena entre o sexo masculino e feminino, contrapondo demais estudos, nos quais as mulheres obtiveram uma maior representatividade na população em estudo [19, 21,22]. 
A crescente feminização dos profissionais da área da saúde pode estar relacionada ao papel tradicional da mulher na história que discorre a respeito da relação de gênero com o dom de cuidar, de educar e de servir [23].

Houve um predomínio de jovens, com faixa etária de até 30 anos, sendo condizente com outros estudos. O fato pode estar relacionado ao aumento dos cursos de graduação e de vagas, principalmente em instituições particulares nos últimos anos [20,21,24]. Este fenômeno pode também estar associado ao grande número de profissionais formados em instituições privadas de ensino superior. Na enfermagem, a abertura de novos cursos entre os anos de 2004 e 2012 contribui com cerca de $70 \%$ da formação destes profissionais em instituições privadas [19,25]. A prevalência de enfermeiros e médicos pós-graduados reafirma resultados de outros estudos, os quais demonstram uma tendência de atualização e qualificação por meio de cursos de especialização na área da saúde [26]. Além disso, a qualificação da formação de recursos humanos na área da saúde, incentivada pelo Ministério da Saúde, tem sido essencial para a transformação das práticas em saúde e para fortalecer as políticas públicas em saúde [23].

Médicos e enfermeiros com menor tempo de experiência profissional também foi evidenciado em outras pesquisas com cenários parecidos $[8,19,24]$. Jovens profissionais são cada vez mais observados na ESF, o que se justifica pela cobertura crescente desta estratégia, resultando em aumento de vagas de emprego [27] associado às mudanças curriculares dos cursos da saúde, com disciplinas e estágios voltados para a consolidação do SUS [19] e da Atenção Primária à Saúde [28] e salários competitivos no mercado e com valorização profissional [29].

O pouco tempo de atuação dos profissionais nas unidades de APS, nos leva a pensar na alta rotatividade dos mesmos, prejudicando diretamente o vínculo com a comunidade e a continuidade das ações em saúde. Diversos são os fatores associados a isto: condições de trabalho, não capacitação, presença de residentes na rede, meios de contratação, busca para qualificação da mão de obra, transferência para outra ESF ou outro estabelecimento de saúde e afastamentos $[19,20,26,30]$. Vale ressaltar que devido à alta rotatividade observada, tornam-se necessárias estratégias promovidas pelas gestões locais que predisponha de recursos de educação permanente. Talvez, isso minimize, por exemplo, o prejuízo nas ações em saúde de promoção da saúde, prevenção de agravos, detecção e tratamentos precoces e reabilitação. Problemas na assistência pré-natal podem ser visualizados até os anos recentes no Brasil, com a persistência de desigualdades regionais e sociais no cuidado prestado [31]. Problemas como toxoplasmose gestacional e fetal [32], sífilis gestacional e congênita [33] e preenchimento inadequado do cartão [34] são frequentes.

De acordo com os resultados, a consulta de pré-natal não faz parte da rotina da maioria dos enfermeiros, ao contrário dos médicos. Esses resultados corroboram um estudo realizado em todo o Brasil, no qual $75,6 \%$ das consultas de pré-natal eram realizadas apenas pelos médicos [35]. Isso nos leva a pensar que estratégias de gestão, organização e planejamento dos serviços locais possam ter influência nos indicadores e na qualidade dos serviços prestados, não sendo somente responsabilidade individual do profissional.

Menos da metade de enfermeiros e médicos relatou a realização de capacitação sobre pré-natal. Algumas reflexões tornam-se necessárias como, por exemplo, repensar a qualidade da capacitação e as estratégias que são utilizadas durante esse processo de educação. Sugerese que as capacitações teórico-práticas sejam contínuas e que haja a criação de protocolos. Com isso, espera-se uma melhora da interação entre médicos e enfermeiros, colaborando para a realização de consultas alternadas, em conformidade com a recomendação do MS $[15,36,37]$.

Cabe registrar, ainda, que o estudo apresentou a limitação de não conseguir captar a totalidade dos profissionais presentes no cenário estudo, apesar de se tratar de um estudo censitário, obtendo uma taxa de resposta acima de $80 \%$. Além disso, a utilização de questionário estruturado para a coleta de dados teve algumas limitações, como foi o caso da perda de participantes de pesquisa. Os profissionais entrevistados precisavam estar motivados para responder ao questionário, já que o mesmo era longo e demandava um pouco de tempo (em torno de 60 minutos) e concentração. Registra-se, também, que o modo de organizar as respostas das questões (escala de Likert) pode ter simplificado em excesso algum conteúdo que exigiria respostas mais complexas para uma maior compreensão da problemática.

Conclusão

Concluiu-se que os profissionais de saúde, enfermeiros e médicos, que atuam na rede de atenção primária à saúde de uma microrregião de Minas Gerais, são predominantemente 
jovens, do sexo feminino, pós-graduados, com pouco tempo de experiência profissional e de atuação na unidade de trabalho, não capacitados para a realização do pré-natal. Além disso, esses profissionais se auto-avaliaram com bom conhecimento e boa capacidade para realizar o pré-natal.

Novos e complementares estudos serão necessários para entender o impacto de diferentes perfis social-profissional de enfermeiros e médicos no processo de trabalho em saúde, sobretudo na Atenção Primária à Saúde e no cuidado pré-natal.

Agradecimentos

À Tassiana Elena de Souza (in memoriam), por tudo, especialmente pelo empenho, amizade, afeto, companhia e aprendizado diário!

\section{Referências}

1. Fertonani HP, Pires DEP, Biff D, Scherer, MDA. Modelo assistencial em saúde: conceitos e desafios para a atenção básica brasileira. Ciênc Saúde Coletiva 2015;20(6):1869-78. https://doi.org/10.1590/1413-81232015206.13272014

2. Damaceno AN, Bandeira D, Hodali N, Weiller TH. Acesso de primeiro contato na atenção primária à saúde: revisão integrativa. Rev APS 2016;19(1):122-38. https://periodicos.ufjf.br/index.php/aps/article/view/15624

3. Silva SA, Baitelo TC, Fracoll LA. Avaliação da Atenção Primária à Saúde: a visão de usuários e profissionais sobre a Estratégia de Saúde da Família. Rev Latinoam Enferm 2015;23(5):979-87. https://doi.org/10.1590/0104-1169.0489.2639

4. Göttems LBD, Pires MRQM. Para Além da Atenção Básica: reorganização do SUS por meio da interseção do setor político com o econômico. Saúde Soc 2009;18(2):189-98. https://doi.org/10.1590/s0104-12902009000200003

5. Pinto LF, Giovanella L. Do programa à Estratégia de Saúde da Família: expansão do acesso e redução das internações por condições sensíveis à atenção básica. Ciênc Saúde Coletiva 2018;23(6):1903-13. https://doi.org/10.1590/141381232018236.05592018

6. Barros NF, Spadacio C, Costa MV. Trabalho interprofissional e as práticas integrativas e complementares no contexto da Atenção Primária à Saúde: potenciais e desafios. Saúde Debate 2018;42(1):163-73. https://doi.org/10.1590/0103-11042018s111

7. Nora CRD, Zobolib ELCP, Vieira M. Problemas éticos vivenciados por enfermeiros na atenção primária à saúde: revisão integrativa da literatura. Rev Gaúcha Enferm 2015;36(1):112-21. http://dx.doi.org/10.1590/1983-1447.2015.01.48809

8. Vendruscolo C, Trindade LL, Rodrigues OCC, Adamy EK, Bum MLB. Introdutório para equipes de saúde da família: contribuições para o fortalecimento da atenção básica. Rev Enferm UFPE on line 2016; 10(9):3393-400. https://doi.org/10.5205/reuol.957183638-1-SM1009201627

9. Galavote HS, Zandonade E, Garcia ACP, Freitas PSS, Seidl H, Contarato PC et al. O trabalho do enfermeiro na atenção primária à saúde. Esc Anna Nery 2016;20(1):90-98. https://doi.org/10.5935/1414-8145.20160013

10. Santos FAPS, Enders BC, Santos VEP, Dantas DNA, Miranda LSMV. Integralidade e atenção obstétrica no Sistema Único de Saúde (SUS): reflexão à luz da teoria da complexidade de Edgar Morin. Esc Anna Nery 2016;20(4):e20160094. https://doi.org/10.5935/1414-8145.20160094

11. Muniz F, Rocha F, Ramos A, Nunes SF. Assistência de enfermagem no pré-natal de baixo risco na atenção primária. J Manag Prim Health Care 2018;9:e3.

https://doi.org/10.14295/jmphc.v9i0.433

12. Tomasi E, Fernandes PAA, Fischer T, Siqueira FCV, Silveira DS, Thumé E et al. Qualidade da atenção pré-natal na rede básica de saúde do Brasil: indicadores e desigualdades sociais. Cad Saúde Pública 2017;33(3):e00195815. https://doi.org/10.1590/0102-311x00195815

13. Instituto Brasileiro de Geografia e Estatística. IBGE. Censo Demográfico 2010. [citado 2016 Out 9]. Disponível em: http://cidades.ibge.gov.br/xtras/perfil.php?lang=\&codmun=317130\&search=minasgerais/vicosalinfograficos:-informacoes-completas 
14. Morosini MVGC, Fonseca AF, Lima LD. Política Nacional de Atenção Básica 2017: retrocessos e riscos para o Sistema Único de Saúde. Saúde Debate 2018;42(116):1124. https://doi.org/10.1590/0103-1104201811601

15. Brasil. Ministério da Saúde. Protocolos da Atenção Básica: Saúde das Mulheres/ Ministério da Saúde, Instituto Sírio-Libanês de Ensino e Pesquisa - Brasília: Ministério da Saúde; 2016. Disponível em:

http://bvsms.saude.gov.br/bvs/publicacoes/protocolos atencao basica saude mulhere s.pdf

16. Ricci SS. Enfermagem materno-neonatal e saúde da mulher. Traduzido por: Azevedo MF. Rio de Janeiro: Guanabara Koogan; 2015.

17. Moreira IJB, Horta JA, Duro LN, Borges DT, Cristofari AB, Chaves J et al. Perfil sociodemográfico, ocupacional e avaliação das condições de saúde mental dos trabalhadores da Estratégia Saúde da Família em um município do Rio Grande do Sul, RS. Rev Bras Med Fam Comunidade 2016;11(38):1-12. https://doi.org/10.5712/rbmfc11(38)967

18. Moreira MCN, Gomes R, Ribeiro CR. E agora o homem vem?! Estratégias de atenção à saúde dos homens. Cad Saúde Pública 2016;32(4):e00060015. https://doi.org/10.1590/0102-311X00060015

19. Faria MGA, Acioli S, Gallasch CH. Perfil de enfermeiros fluminenses da estratégia de saúde da família participantes de um curso de especialização. Enferm Foco 2016;7(1):52-5. https://doi.org/10.21675/2357-707X.2016.v7.n1.667

20. Mendes CLA. Perfil do profissional médico na Estratégia de Saúde da Família no município do Rio de Janeiro: um modelo em transição [Tese]. Rio de Janeiro: Escola Nacional de Saúde Pública Sergio Arouca; 2015.

21. Conselho Federal de Enfermagem (COFEN). Núcleo de Estudos e Pesquisas de Recursos Humanos em Saúde. Perfil da Enfermagem do Brasil - Relatório Final; 2015. Disponível em: http://www.cofen.gov.br/perfilenfermagem/pdfs/relatoriofinal.pdf

22. Lima EFA, Sousa Al, Primo CC, Leite FMC, Souza MHN, Maciel EL. Perfil socioprofissional de trabalhadores de equipes saúde da família. Revista Enferm UERJ 2016;24(1):e9405. https://doi.org/10.12957/reueri.2016.9405

23. Lombardi MR, Campos VP. A enfermagem no Brasil e os contornos de gênero, raça/cor e classe social na formação do campo profissional. Revista da ABET 2018;17(1):28-46. https://doi.org/10.22478/ufpb.1676-4439.2018v17n1.41162

24. Oliveira MCM, Lima TL, Baluta VH. A formação do profissional enfermeiro, no contexto das reformas de ensino no Brasil. Revista Grifos 2014;36(37):161-86. https://bell.unochapeco.edu.br/revistas/index.php/grifos/article/view/2784/1766

25. Scherer MDA, Oliveira CI, Carvalho WMES, Costa MP. Cursos de especialização em Saúde da Família: o que muda no trabalho com a formação? Revista Interfaces 2016;20(58):691-702. https://doi.org/10.1590/1807-57622015.0020

26. Sousa MST, Brandão IR, Feijão JR. A percepção dos enfermeiros sobre educação permanente em saúde no contexto da estratégia saúde da família de sobral (CE). Revista Interfaces: Saúde, Humanas e Tecnologia 2015;2(7):1-6. https://doi.org/10.16891/2317.434X.143

27. Brasil. Ministério da Saúde. Sistema Único de Saúde. Departamento de Atenção Básica. Passo a passos das ações do Departamento de Atenção Básica; 2015. Disponível em: http://189.28.128.100/dab/docs/portaldab/documentos/passo a passo dab2015.pdf

28. Nóbrega-Therrien SM, Souza PMM, Pinheiro FMC, Castro VS. Formação para a Estratégia Saúde da Família na Graduação em Medicina. Revista Brasileira de Educação Médica 2015;39(1):112-8. https://doi.org/10.1590/19812712015v39n1e02212012

29. Tonelli BQ, Leal APR, Tonelli WFQ, Veloso DCMD, Gonçalves DP, Tonelli SQ. Rotatividade de profissionais da Estratégia Saúde da Família no município de Montes Claros, Minas Gerais, Brasil. RFO UPF 2018;23(2):180-5. https://doi.org/10.5335/rfo.v23i2.8314

30. Giovani MSP, Vieira CM. Longitudinalidade do cuidado diante da rotatividade de profissionais na Estratégia Saúde da Família. RECIIS Rev Eletr Com Inov Saúde 2013;7(4). https://doi.org/10.3395/reciis.v7i4.572

31. Domingues RMSM, Viellas EF, Dias MAB, Torres JA, Theme-Filha MM, Gama SGN et al. Adequação da assistência pré-natal segundo as características maternas no Brasil. 
Rev Panam Salud Pública 2015;37(3):140-7.

https://scielosp.org/pdf/rpsp/2015.v37n3/140-147/pt

32. Contiero AP, Cavalli HO, Marchioro AA, Ferreira EC, Caniatti MCCL, Breganó RM.

Toxoplasmosis: an examination of knowledge among health professionals and pregnant women in a municipality of the State of Paraná. Rev Soc Bras Med Trop

2014;47(2):198-203. https://doi.org/10.1590/0037-8682-0016-2014

33. Domingues RMSM, Leal MC. Incidência de sífilis congênita e fatores associados à transmissão vertical da sífilis: dados do estudo nascer no Brasil. Cad Saúde Pública 2016;32(6):e00082415. https://doi.org/10.1590/0102-311X00082415

34. Santos TMMG, Abreu APSB, Campos TG. Avaliação dos registros no cartão de prénatal da gestante. Rev Enferm UFPE on line 2017;11(supl.7):2939-2945.

https://doi.org/10.5205/reuol.11007-98133-3-SM.1107sup201715

35. Viellas EF, Domingues RMSM, Dias MAB, Gama SGN, Theme FMM, Costa JV. Assistência pré-natal no Brasil. Cad Saúde Pública 2014;30(suppl 1):85-100. https://doi.org/10.1590/0102-311X00126013

36. Nascimento AMR, Silva PM, Nascimento MA, Souza G, Calsavara RA, Santos AA. Atuação do enfermeiro da estratégia saúde da família no incentivo ao aleitamento materno durante o período pré-natal. Revista Eletrônica Acervo Saúde 2019;(21):e667. https://doi.org/10.25248/reas.e667.2019

37. Rodrigues EM, Nascimento RG, Araújo A. Protocolo na assistência pré-natal: ações, facilidades e dificuldades dos enfermeiros da Estratégia de Saúde da Família. Rev Esc Enferm USP 2011;45(5):1041-7. https://doi.org/10.1590/S0080-62342011000500002 